\title{
La position de l'adjectif épithète en français : le poids des mots
}

Anne Abeillé et Danielle Godard

\section{(2) OpenEdition}

12 Journals

Édition électronique

URL : http://journals.openedition.org/rlv/1211

DOI : $10.4000 /$ rlv. 1211

ISSN : 1958-9239

Éditeur

Presses universitaires de Vincennes

Édition imprimée

Date de publication : 1 mai 1999

Pagination : 9-32

ISSN : 0986-6124

\section{Référence électronique}

Anne Abeillé et Danielle Godard, "La position de l'adjectif épithète en français : le poids des mots », Recherches linguistiques de Vincennes [En ligne], 28 | 1999, mis en ligne le 09 septembre 2005, consulté le 21 avril 2019. URL : http://journals.openedition.org/rlv/1211 ; DOI : 10.4000/rlv.1211 


\title{
Anne ABEILLÉ et Danièle GODARD \\ IUF et Université Paris 7, TALANA \& CNRS, Université Lille 3, SILEX
}

\section{LA POSITION DE L'ADJECTIF ÉPITHÈTE EN FRANÇAIS : LE POIDS DES MOTS ${ }^{1}$}

\begin{abstract}
RÉSUMÉ
Nous proposons une analyse de l'ordre des adjectifs épithètes par rapport au nom en français qui dépend crucialement de la notion de Poids syntaxique. Dans notre approche, les adjectifs appartenant à certaines sous-classes sémantiques ou morphologiques sont marqués comme de poids léger, et doivent être antéposés, sauf s'ils sont coordonnés ou modifiés. La notion de légèreté permet aussi de rendre compte de l'impossibilité d'avoir des syntagmes adjectivaux complets (avec des compléments ou des modifieurs syntagmatiques) en position antéposée.
\end{abstract}

\section{MOTS-CLÉS}

Adjectif épithète, déficience syntaxique, français, HPSG, poids, ordre des mots. 
La position relative de l'adjectif et du nom dans le GN constitue l'un des problèmes les plus étudiés de la grammaire du français ${ }^{2}$. On le comprend : les données sont complexes. Par exemple, bien que l'ordre soit plus libre qu'en anglais, tout n'est pas possible, et, bien que certaines différences sémantiques paraissent corrélées avec la position de l'adjectif, cet effet est beaucoup plus élusif ou absent dans d'autres cas. La difficulté vient de ce que l'on a cherché à ramener les positions de l'adjectif et du nom à un facteur unique, et propre à cette configuration. Si l'on considère au contraire le problème comme faisant partie de l'ensemble de la question de l'ordre des mots, la nécessité de trouver un facteur unique et toujours opérant disparaît. Il est, en effet, reconnu que l'ordre met en jeu des facteurs divers, et il n'y a aucune raison pour que la configuration de l'adjectif et du nom fasse appel à des facteurs qui lui seraient propres.

Dans cet article, nous adoptons les hypothèses suivantes concernant l'ordre des mots :

- l'ordre des mots (plus exactement des constituants) n'est pas ramené à un problème de constituance : il n'y a pas de correspondance un-un entre une structure et un ordre. Au contraire, l'ordre est vu comme un domaine propre de la grammaire (dans la lignée de Gazdar et al., 1985) ${ }^{3}$;

- lorsque plusieurs ordres sont possibles, ils sont autorisés en parallèle, dans la grammaire, sans règle de mouvement à partir d'une position initiale ou canonique.

Nous nous intéressons principalement aux contraintes syntaxiques gouvernant la place de l'adjectif épithète, et nous proposons un trait syntaxique (trait de « poids »), capable de différencier les lexèmes entre eux aussi bien que les constructions, et pris en compte par les règles d'ordre. Cette analyse laisse leur place aux autres facteurs, qui peuvent éventuellement intervenir pour déterminer l'ordre des mots dans le GN, mais elle implique que ceux-ci ne sont pertinents que là où la contrainte syntaxique basée sur le trait de poids est satisfaite. De plus, nous modélisons uniquement un système de compétence, où tous les ordres acceptables sont égaux. Des contraintes d'un autre ordre peuvent s'y ajouter pour donner des systèmes de préférence ; par exemple, l'étude sur corpus de Wilmet (1981) met en évidence une préférence dont nous ne tenons pas compte pour la postposition de l'adjectif là où les deux ordres sont possibles.

\section{Généralisations empiriques}

Il est clair que de nombreux facteurs interviennent pour déterminer la position de l'adjectif par rapport au nom, des facteurs pragmatiques ou discursifs (comme l'aspect affectif de l'adjectif, Blinkenberg, 1928, ou la structure informationnelle, Nølke, 1996 ; Delbecque, 1990), prosodiques, ou stylistiques (par exemple, en termes de fréquence d'occurrence pour l'adjectif ou d'écarts par rapport à la norme, Wilmet, 1981). Nous rappelons ici quelques généralisations phonétiques, morphologiques et sémantiques avant de présenter les généralisations syntaxiques dont nous allons rendre compte. 


\subsection{Généralisations phonétiques}

On dit parfois que plus les adjectifs sont courts (en nombre de syllabes) plus ils tendent à être antéposés. Comme le montrent Miller et al. (1997), une telle règle, qui serait contraire à l'indépendance de la syntaxe par rapport à la phonologie, ne tient pas en français : de nombreux adjectifs polysyllabiques sont antéposables (agréable, nombreux, etc.). Une version plus élaborée de la contrainte met en jeu la longueur respective du nom et de l'adjectif : les adjectifs monosyllabiques devraient précéder les noms polysyllabiques, et les adjectifs polysyllabiques suivre les noms monosyllabiques. Là encore, les contre-exemples abondent, même si ce type de préférence prosodique peut être attesté (les adjectifs le plus souvent antéposés dans les relevés de Wilmet (1981) sont monosyllabiques : grand, bon, beau, etc.) :

a. Son survêtement bleu / *bleu survêtement

b. Ses innombrables chiens / chiens innombrables

La seule généralisation phonétique valide en compétence est que les adjectifs antéposés sont sujets à des phénomènes de sandhi obligatoires comme la liaison obligatoire, la dénasalisation (bon ami), ou d'allomorphie (beau/bel), et non les postposés (pour lesquels la liaison n'est que facultative).

\subsection{Généralisations morphologiques}

Les adjectifs dérivés sont massivement postposés. Les adjectifs dérivés de participes présents, et de participes passifs, dans leur grande majorité, apparaissent après le nom (voir (2), Grévisse, 1988; Wilmet, 1981), sauf ceux qui sont intensionnels (voir (3), Waugh, 1977 ; Delbecque, 1990).

a. Une décision attendue / *attendue décision, des jeux interdits / *interdits jeux

b. Des circonstances atténuantes / *atténuantes circonstances, des propositions concurrentes / * concurrentes propositions

Un prétendu chef-cuisinier, un soi-disant médecin, un vrai menteur

Les adjectifs dérivés intensionnels (qui prennent pour argument l'intension du nom ou les ensembles dénotés par le nom aux différents indices-mondes), sont soit obligatoirement antéposés (dénommé, fichu, soi-disant), soit indifférents à l'ordre (présumé, prétendu). Comme les adjectifs non prédicatifs dont ils font partie sont majoritairement préposés en français (voir 1.3), on voit que la généralisation sémantique prédomine sur la généralisation morphologique. La généralisation morphologique est également contredite par le comportement d'adjectifs à valeur affective comme charmant, étonnant, époustouflant, assommant, etc. qui peuvent suivre ou précéder le nom. 
Un sous-ensemble des adjectifs dérivés de nom, ceux que l'on appelle parfois « relationnels », et qui sont équivalents à un complément de la forme de $N$, ou peuvent être paraphrasés par « relatif à $\mathrm{N}$ », doivent aussi être postposés : français, routier, médical, psychologique, etc. (voir Delbecque, 1990). Mais les autres adjectifs dérivés de $\mathrm{N}$ (spectaculaire, exceptionnel, ordinaire) peuvent être antéposés.

Le point de vue morphologique, donc, ne donne accès qu'à des généralisations partielles : une généralisation par défaut pour les dérivés verbaux, et une sous-généralisation pour les dérivés nominaux.

\subsection{Généralisations sémantiques}

Il n'y a pas de propriété sémantique générale qui soit liée de manière parfaitement régulière à l'ordre relatif du $\mathrm{N}$ et du $\mathrm{A}$. Ainsi, les antonymes n'ont pas un comportement identique ; par exemple, beau est antéposable, tandis que moche ou laid sont toujours postposés (voir Grevisse, 1988 ; Wilmet, 1981). De même, pour les adjectifs mobiles, il est difficile de trouver un effet de sens régulièrement associé à leur position : beaucoup d'adjectifs sont à la fois antéposables et postposables, avec le même sens (en termes vériconditionnels) ${ }^{4}$.

Sans entrer dans le problème difficile de la sémantique des adjectifs, nous nous appuyons sur certaines classes connues. D'abord, nous reprenons la distinction de Kamp (1975) entre adjectifs prédicatifs et non prédicatifs, qui éclaire le comportement positionnel des $\mathrm{A}^{5}$ : les adjectifs non prédicatifs sont plutôt antéposés, et les adjectifs prédicatifs sont plutôt postposés. Les prédicatifs dénotent un prédicat du premier ordre (un ensemble d'entités du domaine), ce que ne font pas les A non prédicatifs (qui ont des sémantiques diverses) ${ }^{6}$. On distingue au moins trois classes de non prédicatifs :

- les quantifiants (ordinaux, cardinaux, indéfinis) sont toujours antéposés ;

- les intensionnels (ancien, vrai, futur dans un ancien coffre, une vraie montagne, un futur champion) sont en général antéposés, mais certains, dérivés de participe, sont indifférents (voir 1.2.) ;

- les subsectifs, pour lesquels la combinaison avec le $\mathrm{N}$ donne un sousensemble de l'ensemble dénoté par le $\mathrm{N}$, selon une norme définie en fonction de l'objet dénoté par le nom ${ }^{7}$, sont antéposés pour les plus fréquents (grand, gros, petit), et ont les deux ordres pour les autres (excellent, minuscule, immense, médiocre).

Les adjectifs prédicatifs (également appelés intersectifs), qui dénotent un ensemble par eux-mêmes et pour lesquels la combinaison avec le $\mathrm{N}$ donne l'intersection des deux ensembles, se divisent en classifiants et non classifiants ${ }^{8}$. Les intersectifs classifiants sont postposés (comme ceux dénotant une couleur, une forme, une substance, une origine...) ${ }^{9}$ : rond, carré, caillouteux... Les intersectifs non classifiants ont généralement les deux ordres (fragile, affreux, agréable, joli...), même si certains sont obligatoirement postposés (moche). 
En plus de cet emploi comme modifieur (prédicatif ou non prédicatif), les adjectifs épithètes dérivés d'un nom (présidentiel, pétrolier, français...) peuvent être arguments de $\mathrm{N}$ : le refus allemand est équivalent à le refus des Allemands, la production pétrolière est équivalent à la production de pétrole. Ils sont obligatoirement postposés ${ }^{10}$.

Il a été souvent observé que certaines formes ont un sens différent suivant qu'elles apparaissent devant ou après le nom ${ }^{11}$. Considérons les exemples suivants :
a. Un ancien coffre $v s$ un coffre ancien
b. Un sacré travailleur vs l'autel sacré de la patrie
c. Un gros fumeur vs un fumeur gros

Dans l'exemple (a), le A préposé est intensionnel, alors que le A postposé est intersectif. Dans l'exemple (b), le A préposé est subsectif (il équivaut à " extrêmement bon »), et le A postposé intersectif classifiant. Enfin, dans l'exemple (c), le A préposé est toujours subsectif, nous semble-t-il, mais l'ensemble de référence n'est pas le même ; il s'agit de l'ensemble des fumeurs dans un cas, et de l'ensemble des hommes dans l'autre cas. En fait, les paires souvent citées du type (4c) sont trompeuses, car l'antéposition est compatible avec les deux interprétations. Avec un certain nombre de ces A, l'antéposition est la position normale, lorsqu'ils sont employés nus, sans modifieur et sans accent contrastif : un gros chien/?? un chien gros, un grand adolescent/?? un adolescent grand. Il est possible que le A postposé en (4c) soit en fait accentué, ou que la postposition ne paraisse naturelle que dans la mesure où l'on construit un contraste avec l'autre interprétation, qui ne semble disponible, pour cet $\mathrm{A}$, que dans l'antéposition ${ }^{12}$.

Malgré les hésitations de certains, il est clair qu'on doit dans certains cas distinguer entre deux lexèmes : même si l'on a le sentiment que la relation sémantique dénotée par l'adjectif a quelque chose de commun dans les deux occurrences, l'argument de l'adjectif n'est pas le même selon que l'adjectif est intensionnel ou non. Ainsi, un ancien coffre n'est pas un coffre ancien, ce n'est pas même un coffre dans la situation de référence, mais quelque chose qui a été un coffre dans une autre situation ou monde ; on distingue donc ancien 1, intensionnel et préposé de ancien 2 intersectif classifiant et postposé. De même, nous aurons deux adjectifs sacré, vrai, ancien, simple dont l'un (intensionnel) est spécifié lexicalement comme devant apparaître devant le nom et l'autre (intersectif) comme devant apparaître après. Le cas de gros, grand, bon, etc. est moins clair. On peut concevoir deux homonymes avec des arguments différents, ou un A sous-spécifié, susceptible de s'appliquer à différentes facettes du N.

En considérant les deux grands groupes de prédicatifs et non prédicatifs, on pourrait penser à proposer des généralisations par défaut. Ce n'est, en fait, pas intéressant, étant donné le positionnement libre des A intersectifs non-classifiants, d'une part, et les contraintes qui constituent des sous-généralisations, d'autre 
part : les A argumentaux et les intersectifs classifiants suivent le $\mathrm{N}$; si on croise la morphologie et la sémantique, on peut ajouter que les intensionnels non dérivés de $\mathrm{V}$ précèdent le $\mathrm{N}$.

\subsection{Généralisations syntaxiques}

En français, les généralisations syntaxiques concernant les adjectifs mobiles sont les suivantes :

(i) Les adjectifs apparaissent après le $\mathrm{N}$ s'ils ont des compléments (Blinkenberg, 1928) :

a. Un long entretien / un entretien long

b. * Un long de deux heures entretien / un entretien long de deux heures

a. Une facile victoire / une victoire facile

b. * Une facile à remporter victoire / une victoire facile à remporter

(ii) Les adjectifs prénominaux peuvent être modifiés par certains adverbes (les modifieurs de degré dans l'ensemble, très, trop, assez, vraiment, peu), mais d'autres modifieurs adverbiaux (absolument, véritablement) et tous les modifieurs syntagmatiques les rendent obligatoirement postnominaux :

(7) a. Une vraiment belle jeune fille / Une jeune fille vraiment belle

b. Une (*véritablement) belle jeune fille / Une jeune fille (véritablement) belle

(8) a. Une décision (très) habile / Une (très) habile décision

b. Une (*politiquement) habile décision / Une décision (politiquement) habile

a. Un plus long (*que prévu) entretien / Un entretien plus long que prévu

b. Cette étrange (*à vos yeux) décision / Cette décision étrange (à vos yeux)

(iii) Les adjectifs prénominaux peuvent être lexicalement coordonnés, mais pas par des conjonctions discontinues ou initiales :

(10) a. Une belle et longue table / une table belle et longue

b. * Une et belle et longue table / une table et belle et longue

c. Une (*à la fois) belle et longue table / une table à la fois belle et longue

Un fait frappant concerne les adjectifs obligatoirement prénominaux. Certains intensionnels, quand ils sont coordonnés, deviennent postposables :

(11) a. Des faux coupables / $*$ Des coupables faux

b. Des vrais coupables / *Des coupables vrais

c. Des vrais ou faux coupables / Des coupables vrais ou faux

(12) a. Les anciens sénateurs / *Les sénateurs anciens

b. Les actuels sénateurs / ?Les sénateurs actuels

c. Les anciens ou actuels sénateurs / Les sénateurs anciens ou actuels 
De la même façon, certains subsectifs, préposés quand ils sont seuls, acquièrent la possibilité d'apparaître après le $\mathrm{N}$ quand ils sont coordonnés ou modifiés (Blinkenberg, 1928) :

(13) a. Un grand avantage / ?*Un avantage grand

b. Un plus grand avantage / Un avantage plus grand

(14) a. Un bon poulet / ?*Un poulet bon

b. Un bon et gros poulet / Un poulet bon et gros

Les autres généralisations syntaxiques concernant les adjectifs postnominaux sont les suivantes :

(iv) Ils permutent avec les GP compléments (qui sont eux-mêmes permutables) ${ }^{13}$ :

(15) a. Une robe superbe de Dior / Une robe de Dior superbe

b. Une participation impressionnante à la conférence / Une participation à la conférence impressionnante

(16) a. Un livre intéressant de Mauss sur les Indiens / de Mauss intéressant sur les Indiens / de Mauss sur les Indiens intéressant

b. Une lettre très aimable de Paul à son directeur / ?de Paul très aimable à son directeur / de Paul à son directeur très aimable

(v) Ils peuvent modifier un $\mathrm{N}$ ou un GN (ils ont portée large sur une coordination de $\mathrm{N}$ et peuvent avoir portée large sur une coordination de GN) :

(17) a. Les officiers et sous-officiers distingués

b. Des projets d'autoroutes et des chantiers impressionnants (ambigu)

A contrario, l'adjectif prénominal peut avoir portée large sur une coordination de (certains) $\mathrm{N}$ (pluriels), mais pas de $\mathrm{N}$-barre ( $\mathrm{N}$ avec leurs compléments) ${ }^{14}$ :

(18) a. Les anciens officiers et sous-officiers

b. Les meilleurs étudiants ou étudiantes de la classe

(19) a. Il a tourné la page sans grande peine de cœur ou haine de soi

b. C'est un endroit idéal pour vaillants pêcheurs de truite et amoureux de la nature

En (19a) c'est la peine de cœur qui est grande, non la haine de soi ; en (19b), la seule interprétation est que les pêcheurs soient vaillants (non les amoureux).

(vi) Les incises sont possibles entre le $\mathrm{N}$ et l'adjectif postposé, non entre l'adjectif antéposé et le $\mathrm{N}$ :

(20) a. * Une élégante, si j'ose dire, poubelle

b. Une poubelle, si j'ose dire, élégante 
L'adjectif épithète antéposé est donc adjacent au N, tandis que l'épithète postposé est plus mobile. En termes structuraux, l'adjectif antéposé est toujours adjoint au $\mathrm{N}$, tandis que l'épithète postposé est soit au même niveau que les compléments de N (avec lesquels il permute), soit adjoint au GN. Si l'on reprend la distinction (à vocation universelle) proposée par Sproat et Shih (1988) entre modification directe et indirecte, on peut dire que l'épithète antéposé est toujours un modifieur direct, tandis que l'épithète postposé a les deux possibilités ${ }^{15}$. Enfin, la combinaison entre l'A antéposé et le $\mathrm{N}$ est de type lexical (l'A antéposé ne peut projeter un syntagme adjectival complet) tandis que celle entre l'A postposé et le $\mathrm{N}$ est de type syntagmatique. Nous proposons de corréler ces deux types de contraintes (projection ou non d'un syntagme, adjacence au $\mathrm{N}$ tête ou non) grâce à la notion de « légèreté ».

\section{Ordre et trait de poids}

Nous nous appuyons sur la théorie plus générale de l'ordre des mots développée dans Abeillé et Godard (1998b = A \& G), et qui repose sur un trait de poids, à deux valeurs (léger et non léger), qui différencie entre eux les lexèmes et les syntagmes. Dans notre optique, le poids résulte d'une part d'une spécification lexicale (certains adjectifs épithètes sont légers, d'autres non), d'autre part des règles de construction des syntagmes (certains types de syntagmes sont non légers, d'autres pas).

\subsection{Données parallèles dans le GV}

Si l'on considère la position relative de l'adjectif et du $\mathrm{N}$ tête comme un problème d'ordre, on s'attend à ce que des données comparables soient mises à jour dans d'autres domaines que le GN. Le GV offre ainsi des données intéressantes :

(i) Certains adverbes (bien, mal, mieux, trop, beaucoup, vraiment, etc.) peuvent apparaître à gauche du V lexical (infinitif) sans pouvoir avoir portée large sur une coordination de GV :

a. Jean essaie de mieux lire.

b. Jean voulait [mieux lire le texte et l'expliquer aux élèves]

(21b) dit que Jean veut lire mieux le texte et l'expliquer aux élèves, non qu'il veut aussi l'expliquer mieux. On admet (Abeillé et Godard, 1997 et A \& G) que ces adverbes sont adjoints à $\mathrm{V}$ (et non à $\mathrm{GV}$ ). Lorsque ces adverbes sont modifiés par les adverbes de degré très, beaucoup, assez, vraiment, etc., ils peuvent précéder le $\mathrm{V}$ infinitif lexical, mais non lorsqu'ils sont modifiés par d'autres adverbes ou syntagmes : 
(22) a. Jean a réussi à très bien lire le texte.

b. * Jean a réussi à incroyablement bien lire ce texte

c. Jean a réussi à lire ce texte incroyablement bien.

a. Jean essayait de mieux travailler / travailler mieux

b. * Jean essayait de mieux que toi travailler

c. Jean essayait de travailler mieux que toi.

Ces mêmes adverbes peuvent être coordonnés, en position préverbale, mais doivent être postposés en cas de coordination marquée par une conjonction discontinue :

(24) a. Il avait peur de trop ou trop peu travailler.

b. ?* Il avait peur de soit trop soit trop peu travailler

c. Il avait peur de travailler soit trop soit trop peu.

On a donc un parallèle entre les propriétés de ces adverbes et les modifications et coordinations autorisées pour les adjectifs prénominaux.

(ii) Ces mêmes adverbes peuvent être aussi postverbaux, avec des restrictions positionnelles : ils doivent précéder les compléments syntagmatiques, sauf s'ils sont modifiés ou coordonnés ${ }^{16}$ :

(25) a. Jean lit bien ce texte / ??lit ce texte bien

b. Paul aime beaucoup son frère / *son frère beaucoup

(26) a. Jean lit très bien ce texte / lit ce texte très bien

b. Jean lira bien ou mal ce texte / lira ce texte bien ou mal

c. Paul aime vraiment beaucoup son frère / lit. aime son frère vraiment beaucoup

Ces contraintes se retrouvent avec les compléments nominaux sans déterminant (« nus »), qui doivent précéder les compléments syntagmatiques, sauf s'ils sont modifiés ou coordonnés ${ }^{17}$ :

(27) a. La chaleur donne soif à Jean / *donne à Jean soif

b. Ce livre fait peur à Marie / *fait à Marie peur

(28) a. La chaleur donne faim et soif à Jean / à Jean faim et soif

b. Ce livre fait envie et peur à Marie / fait à Marie envie et peur

c. La chaleur donne vraiment soif à Marie / à Marie vraiment soif

Tout se passe comme si ces adverbes avaient une contrainte d'adjacence (ou plutôt de proximité) à la tête verbale.

(iii) Les autres adverbes sont plus libres dans le $\mathrm{GV}^{18}$ : ils suivent la tête verbale et les compléments nus, et permutent avec les compléments syntagmatiques, rappelant en cela les adjectifs post-nominaux ${ }^{19}$ :

(29) a. Paul lit un livre à sa grand-mère gentiment.

b. Paul lit gentiment un livre à sa grand-mère.

c. Paul lit un livre gentiment à sa grand-mère. 
Explicitons le parallélisme. Le contraste entre le comportement des adverbes de (i) suivant qu'ils sont ou non coordonnés et modifiés rappelle évidemment celui des adjectifs « nus » obligatoirement prénominaux. La liberté des autres adverbes (et des compléments syntagmatiques) rappelle la liberté des adjectifs postnominaux. Une bonne analyse doit rendre compte de cette communauté de comportement dans le GN et le GV.

\subsection{Constituants légers et non légers}

Notre analyse peut être résumée de la façon suivante. La description des lexèmes et des syntagmes comporte le trait syntaxique POIDS qui a deux valeurs, léger et non léger ${ }^{20}$. Les lexèmes sont lexicalement spécifiés comme légers ou non légers, ou sont sous-spécifiés. La plupart des syntagmes sont non légers, seule la coordination de mots légers ou la modification d'un mot léger par un mot léger peut être de poids léger. En simplifiant les règles d'ordre, on peut dire que les constituants légers précèdent les non légers. Les adjectifs épithètes qui précèdent le $\mathrm{N}$ tête sont légers, ceux qui le suivent sont non légers.

Notre analyse prend place dans la construction d'une grammaire du français dans le modèle syntagmatique HPSG ${ }^{21}$, qui a les caractéristiques suivantes :

(a) les objets linguistiques (mots, syntagmes) sont décrits par des ensembles de traits structurés et typés ; chaque sous-type hérite des descriptions associées au type ;

(b) le modèle est surfaciste (un seul niveau de représentation) ;

(c) l'unité syntaxique minimale est le mot ; il n'y a donc pas d'unité syntaxique autonome correspondant à une «catégorie fonctionnelle » de la grammaire transformationnelle (sauf si cette dernière se trouve coïncider avec un mot dans une langue) ;

(d) les contraintes d'ordre sont séparées de la dominance ; elles contraignent les suites de constituants de même niveau dans un syntagme.

Considérons les lexèmes. Selon les données de 2.3. et 2.4., il est clair que la position de l'adjectif par rapport au $\mathrm{N}$ dépend partiellement des propriétés lexicales, que nous représentons par le trait de poids. Les adjectifs qui apparaissent obligatoirement devant le $\mathrm{N}$ lorsqu'ils sont « nus » (adjectifs non prédicatifs) sont lexicalement [POIDS léger], les adjectifs qui sont toujours postnominaux sont [POIDS non léger], et les autres adjectifs qui peuvent apparaître devant comme après le $\mathrm{N}$, ne sont pas lexicalement spécifiés quant à leur poids ${ }^{22}$. Les adverbes se divisent en [POIDS léger] et [POIDS non léger]. Ceux qui peuvent s'adjoindre à gauche de l'infinitif lexical, et qui, en emploi postverbal, doivent précéder les compléments syntagmatiques, sont légers (bien, mal, beaucoup, peu, à peine, etc.) ; les autres adverbes sont non légers. De même, les $\mathrm{N}$ se divisent en légers et non légers, puisque les $\mathrm{N}$ communs sont légers, alors que les $\mathrm{N}$ propres (employés sans déterminant) sont non légers. Les verbes, dans l'ensemble, sont sous-spécifiés. 
Pour les données examinées ici, trois types de syntagmes sont nécessaires : le syntagme tête-compléments-ajouts, le syntagme tête-ajout, et le syntagmecoordonné ${ }^{23}$. Nous donnons en (30) les structures de traits associées aux deux premiers ${ }^{24}$ :

a. Syntagme tête-ajout :

$\left[\begin{array}{l}\text { B-TETE [SYNSEM } 1] \\ \text { B-NON-TETE }<[\text { MODIF } 1]\end{array}\right]$

b. Syntagme tête-compléments-ajouts :

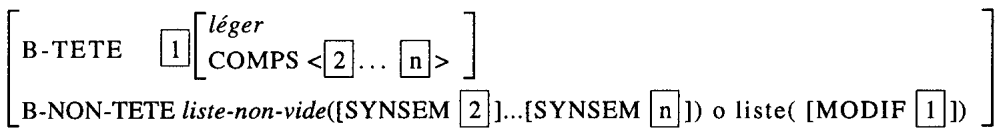

Le syntagme tête-ajout est illustré par les exemples suivants : longue table, très longue, bien manger, [une table et une chaise] magnifiques. Il comporte deux branches et contraint la branche non-tête (ou ajout) à avoir un trait MODIF dont la valeur indique les traits syntaxiques et sémantiques (regroupés en SYNSEM) de la catégorie modifiée (la tête). Le syntagme tête-compléments-ajouts comprend une branche tête (qui doit être « légère ») d'une part, et une liste (non vide) de branches compléments, et une liste d'ajouts optionnels (qui doivent chacun avoir un trait Modif à valeur compatible avec les traits de la tête modifiée), d'autre part. Ce schéma rend compte des permutations observées entre compléments et ajouts en les introduisant au même niveau comme frères de la tête $(\mathrm{N}$ ou $\mathrm{V})$ [voir aussi Kasper 1994]. Ainsi, les syntagmes lira bientôt Proust à ses étudiants ou (une) conception stupide de la passion sont des instances de ce type.

Dans l'ensemble, les syntagmes sont non légers. Cette contrainte est aisée à formuler si l'on s'appuie sur le typage des syntagmes (ou constructions ; voir Sag, 1997). Une distinction majeure existe entre syntagme-avec-tête et syntagmesans-tête (dont le syntagme coordonné) ; les premiers se divisent entre tête-ajout et tête-nexus (dont les sous-types sont les autres syntagmes avec tête). Puisque les seuls syntagmes à ne pas être obligatoirement non légers sont le syntagmecoordonné d'une part, et le tête-ajout d'autre part, on exprime la contrainte comme en (31), qui dit que le type tête-nexus implique la propriété d'être non léger :

$$
\text { syntagme-tête-nexus }=>\text { [POIDS non léger }]
$$

La possibilité pour les syntagmes coordonnés et tête-ajout d'être légers ou non légers est également formulée comme une contrainte implicationnelle sur les types ${ }^{25}$ : 
a.

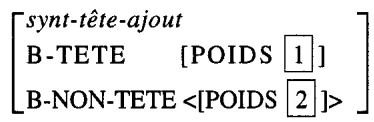$$
\Rightarrow[\text { POIDS }(1 \cup 2) \vee \text { non-léger }]
$$

b. $\left[\begin{array}{l}\text { synt-coord } \\ \text { B-NON-TETE liste([POIDS } 1, \ldots \text { POIDS }[\mathrm{n}])\end{array}\right] \Rightarrow$ [POIDS $\left(\begin{array}{lll}1 & \ldots \cup[\mathrm{n}) \vee \text { non-léger }]\end{array}\right.$

Dans les deux cas, le poids du syntagme, donné à droite de l'implication, est fonction du poids des branches, donné à gauche, ou non léger. En (32a), le poids de la tête est représenté par 1 et celui de la branche non-tête (l'ajout) est représenté par $2 . \mathrm{Si} 1$ et 2 sont identiques, ils peuvent s'unifier : s'ils sont légers, le syntagme est léger ou non léger (donc sous-spécifié) ; s'ils sont non légers, le syntagme est non léger. S'ils sont de poids différents, l'unification échoue $(1 \cup 2$ ne correspond à rien) et le syntagme est non léger. Pour les syntagmes coordonnés, les branches forment une liste de constituants qui sont chacun léger ou non léger. Comme précédemment, les poids des branches s'unifient s'ils sont identiques, et ne s'unifient pas s'ils ne le sont pas (la première valeur ne correspond à rien). Donc, si toutes les branches sont légères, le syntagme est léger ou non léger (sous-spécifié) ; si toutes les branches sont non légères, ou si l'une est non légère, le syntagme est non léger.

Prenons quelques exemples. En tant que syntagme tête-complémentsajouts (la liste des compléments n'est pas vide, mais les ajouts ne sont pas obligatoires), le GV lit Proust à ses étudiants est non léger; il en va de même pour le syntagme formé d'un adjectif et de ses compléments, comme facile à remporter. Puisque le syntagme tête-ajout est non léger dès que l'un des deux constituants est non léger, le syntagme mange rapidement est non léger, comme le syntagme incroyablement belle, où les adverbes rapidement et incroyablement sont non légers ; mais les syntagmes bien manger, très belle, vraiment très belle peuvent être légers ou non légers, suivant le contexte. Les coordinations d'adjectifs légers intensionnels comme vrais ou faux sont a priori sousspécifiées ; en contexte, elles sont légères à gauche du $\mathrm{N}$ et non légères à droite du N (voir les contraintes d'ordre en 2.3). De même, la coordination de deux V légers, lit et commente, est sous-spécifiée et instanciée comme légère dans lit et commente Proust, en vertu de la contrainte sur le poids de la tête du syntagmetête-compléments-ajouts ${ }^{26}$.

\subsection{L'adjectif épithète}

On distingue les adjectifs lexicalement selon leur poids : ceux qui sont obligatoirement prénominaux sont légers, ceux qui sont obligatoirement postnominaux sont non légers, les autres (la majorité des adjectifs en français) 
sont sous-spécifiés ${ }^{27}$. Le poids de l'adjectif interagit dans le GN avec la constituance d'une part, avec l'ordre, d'autre part. Les adjectifs épithètes, ou modifieurs, en tant qu'ajouts, comportent dans leur description un trait MODIF dont la valeur indique les traits de la catégorie à laquelle ils peuvent s'adjoindre (c'est ainsi qu'est pris en compte l'accord). Les adjectifs légers (employés nus) ne peuvent s'adjoindre qu'à un $\mathrm{N}$ léger, tandis que les adjectifs non légers s'adjoignent à $\mathrm{N}$ ou GN.

Deux illustrations de l'adjonction des adjectifs légers au N léger sont ainsi données en (34), où les branches de l'arbre portent l'annotation de leur fonction (T pour tête, SPR pour spécifieur, AJT pour ajout, COMP pour complément et CONJ pour membre d'une coordination) :
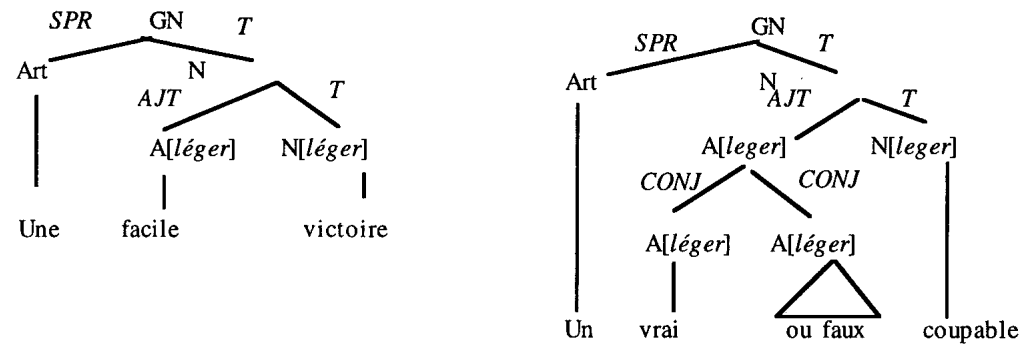

De leur côté, les adjectifs non légers peuvent s'adjoindre au N léger ou non léger. Si l'on accepte nos hypothèses sur la constituance, un $\mathrm{N}$ lexical (ou modifié par un adjectif léger) se combine avec ses compléments, et les compléments et les ajouts permutent en étant au même niveau de structure (voir supra). D'autre part, l'adjectif à droite peut avoir portée large sur une coordination de syntagmes, ce qui montre que l'adjectif peut modifier un $\mathrm{N}$ non léger (voir supra). On ne spécifie donc pas le poids du $\mathrm{N}$ auquel les adjectifs non légers s'adjoignent, ce qui laisse ouvertes les deux configurations. Les A non légers autorisés par le syntagme têtecompléments-ajouts et le syntagme tête-ajout sont illustrés en (35) et (36) :

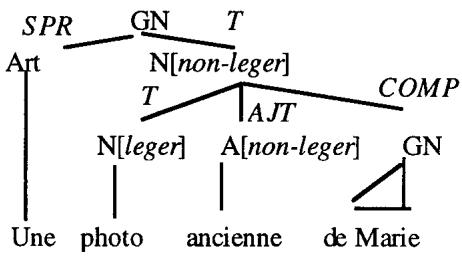


(36)

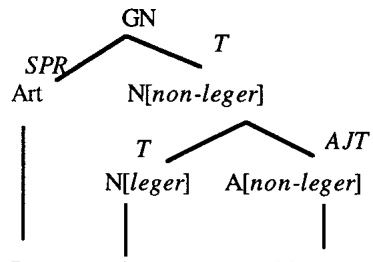

Des conséquences inquiétantes

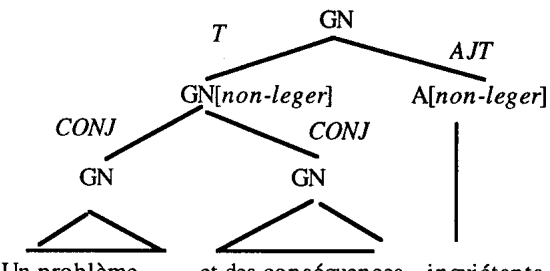

Un problème et des conséquences inquiétants

On aboutit à la classification croisée de (37), pour les mots et syntagmes pertinents pour nos exemples, selon leur poids :

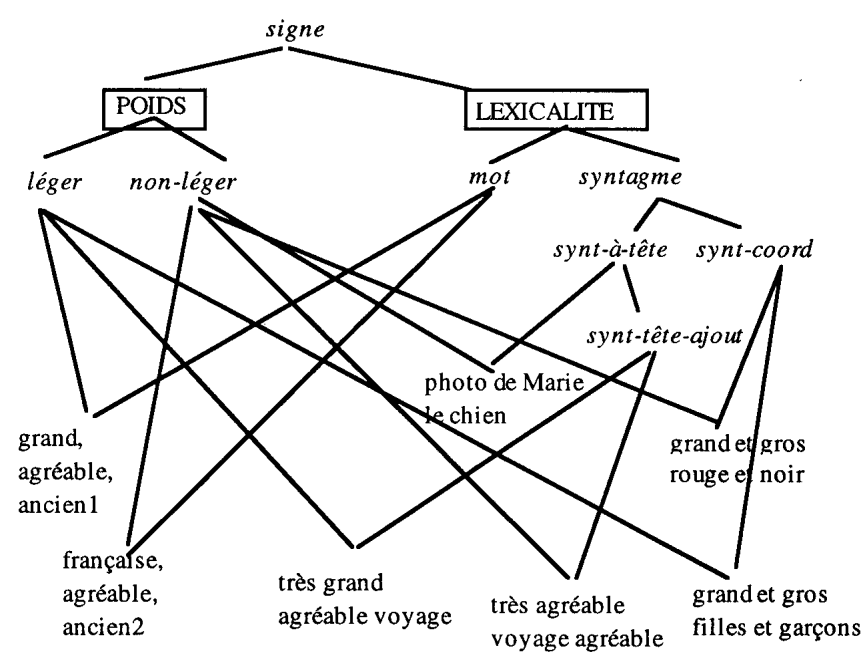

Nous nous tournons finalement vers les contraintes d'ordre qui sont pertinentes pour les adjectifs dans le GN ${ }^{28}$. Prenons d'abord le syntagme tête-ajout. La contrainte (38a) ordonne l'ajout léger avant la tête, que cette dernière soit ou non légère :

(38) Contraintes d'ordre sur le syntagme Tête-Ajout

a syntagme-tête-ajout $=>$ non-Tête [léger] $<$ Tête

b. $\quad$ syntagme-tête-ajout $=>$ Tête [léger ou nom] $<$ non-Tête [non léger]

La contrainte (38a) suffit à imposer que l'adjectif léger précède le $\mathrm{N}$ tête du GN. De même, elle ordonne les adverbes légers avant la tête qu'ils modifient, par exemple le A (très beau vs * beau très), le V lexical (bien manger, très bien manger, bien ou mal manger) ${ }^{29}$. La contrainte (38b) ordonne non seulement les ajouts non légers après les têtes légères, d'une manière générale, mais également 
après les têtes non légères si elles sont de la catégorie N. Ceci permet d'obtenir les adjectifs non légers et frères du $\mathrm{N}$ lexical tête après le $\mathrm{N}$, mais aussi après le GN (comme en (36)) ; il en va de même pour les relatives, qui ne peuvent pas apparaître avant le GN. Les ajouts au $\mathrm{N}$ se distinguent des ajouts aux catégories verbales, puisque ces derniers, s'ils sont non légers, précèdent ou suivent les GV et les Phrases (ou V non légers).

Reste l'ordre dans le syntagme tête-compléments-ajouts. D'une part, la tête lexicale (ou modifiée par un ajout léger) vient en premier (39a). D'autre part, les ajouts et les compléments non légers ne sont pas ordonnés entre eux : ils ne demandent aucune contrainte d'ordre supplémentaire. Mais, on l'a vu en 2.1 avec les données du GV, il en faut une pour ordonner les compléments légers avant les autres ; c'est ce que fait (39b) ${ }^{30}$.

(39) Contraintes d'ordre sur le syntagme tête-compléments-ajouts

a. syntagme-tête-compléments-ajouts $=>$ Tête $<$ non-têtes

b. syntagme-tête-compléments-ajouts $=>$ [léger $]<$ [non léger $]$

On illustre ci-après quelques exemples de linéarisation.

\begin{tabular}{|l|l|l|l|}
\hline a. & Une & facile & victoire \\
\hline & & AJOUT [léger] & TÊTE [léger] \\
\hline b. & Une & victoire & facile \\
\hline & & TÊTE [léger] & AJOUT [non léger] \\
\hline c. & Une & victoire & facile à remporter \\
\hline & & TÊTE [léger] & AJOUT [non léger] \\
\hline d. * & Une & facile à remporter & victoire \\
\hline & & AJOUT [non léger] & TÊTE [léger] \\
\hline
\end{tabular}

\begin{tabular}{|l|l|l|l|}
\hline a. & Des & faux & coupables \\
\hline & & AJOUT [léger] & TÊTE [léger] \\
\hline b. $*$ & Des & coupables & faux \\
\hline & & TÊTE [léger] & AJOUT [léger] \\
\hline c. & Des & vrais ou faux & coupables \\
\hline & & AJOUT [léger] & TÊTE [léger] \\
\hline d. & Des & coupables & vrais ou faux \\
\hline & & TÊTE [léger] & AJOUT [non léger] \\
\hline
\end{tabular}




\section{Comparaison avec d'autres approches}

Nous comparons maintenant notre proposition à d'autres analyses concernant la position de l'adjectif dans le GN ou des distinctions plus générales pertinentes pour l'ordre des mots.

\subsection{Analyse de l'ordre de $\mathbf{N}$ et $\mathrm{A}$}

Considérons d'abord les contraintes syntaxiques gouvernant les adjectifs antéposés. On les retrouve en anglais, en italien, et plus généralement dans les langues SVO (ou à verbe initial) : les syntagmes adjectivaux dont la tête est un adjectif antéposé ou antéposable ne sont pas antéposables, sauf s'ils mettent en jeu une modification ou une coordination « légère ». Pour rendre compte de l'impossibilité pour les adjectifs anglais d'apparaître devant le $\mathrm{N}$ lorsqu'ils sont accompagnés de leurs compléments en anglais, Williams (1981) propose un filtre tel que le dernier constituant des A prénominaux doit être la tête. Ce filtre est insuffisant pour l'anglais : il prédit qu'on ne pourra avoir A fair enough proposal (puisque fair précède son modifieur dans le GA) et qu'on aura *A so important decision, puisque important est bien final dans le GA (alors qu' on a seulement $A$ decision so important). Il ne dit rien des syntagmes coordonnés. De plus, cette proposition n'a pas l'ambition de dire quelque chose de l'ordre des mots en général, et ne peut expliquer l'ordre relatif de l'Aet du $\mathrm{N}$ en français.

Les travaux récents concernant la position du A épithète se divisent en deux groupes. La première approche (Cinque, 1990 ; Rouveret, 1994) met en avant l'hypothèse que les différences sont dues, tant à l'intérieur d'une langue qu'au travers des langues, à la position du N (qui se déplace) dans une structure caractérisée par la présence de projections fonctionnelles. Les A, quant à eux, ne changent pas : ils sont modifieurs (ou spécifieurs) de ces projections, de manière appropriée à leur sémantique. La deuxième approche met l'accent sur la différence entre la modification à gauche et à droite du N. Sproat et Shih (1988) opposent la « modification directe » du A prénominal à la modification indirecte du A qui suit le $\mathrm{N}$ (semblable à celle d'une relative), avec quantité de données dans des langues diverses à l'appui de cette distinction. De manière intéressante, le $\mathrm{A}$ à gauche du $\mathrm{N}$ est pour eux une adjonction d'un $\mathrm{A}$ lexical à un $\mathrm{N}$ lexical, et le résultat est lui aussi lexical, alors que la combinaison avec un $\mathrm{A}$ à droite du $\mathrm{N}$ donne un syntagme. Pour Bouchard (1998), le A lexical est adjoint à gauche du N lexical (ils forment un syntagme), alors que le A lexical ou syntagmatique s'adjoint à droite du GN. L'analyse de Valois (1991) est intermédiaire. Il adopte, pour le A à gauche du N la même analyse que Sproat et Shih, renommant le mode de combinaison « incorporation » (sans donner d'explications techniques sur ce qu'il entend par là), mais adopte, pour le $\mathrm{A}$ à droite du N, le même type de solution que dans la première approche.

Notre analyse et notre choix général de représentation sont éloignés de la première approche qui exprime en termes de structure ce que nous disons en termes d'ordre. Une telle analyse est sans doute plus propre à rendre compte des 
différentes inter-langues que du détail des données dans une langue comme le français. On ne voit pas bien pourquoi il y aurait une différence entre les A suivant qu'ils sont ou non coordonnés et modifiés (pour les A intensionnels), ou suivant qu'ils ont ou non des compléments (pour les A intersectifs mobiles).

Notre analyse est plus proche du second type. En effet, notre hypothèse d'un « syntagme léger » lorsque le A léger s'adjoint à gauche du N léger représente bien la même analyse que la « modification directe ». Quant au statut syntaxique de ces constructions légères, nous avons choisi de les présenter comme des syntagmes parce qu'ils font appel à des processus qui ont cours en syntaxe (adjonction, coordination). Mais on pourrait aussi considérer que de telles combinaisons existent en morphologie ${ }^{31}$. Ce qui manque cependant aux analyses de Sproat et Shih et de Valois, c'est le moyen de régler la répartition des A : comment empêcher les A toujours postposés de s'incorporer au $\mathrm{N}$, et comment forcer ceux qui sont toujours à gauche à le faire ? Par exemple, forcer l'incorporation revient à retirer à la forme le statut de mot indépendant. Comment alors, cette forme peut-elle acquérir ce statut par la modification ou la coordination ? Il est nécessaire en fait de faire appel à une distinction qui peut, d'une part, faire la différence entre les items lexicaux, et d'autre part, rendre compte de la différence entre items lexicaux nus et syntagmes (présence de compléments) ou combinaisons légères.

\subsection{Distinctions pertinentes pour l'ordre}

Notre distinction en termes de poids est pertinente pour l'ensemble des problèmes d'ordre. Elle est alors à rapprocher d'autres propositions, avancées pour d'autres phénomènes, et qui visent à rendre compte de ce que certaines expressions sont plus contraintes que d'autres en ce qui concerne l'ordre. Sells (1994), à partir de données du Coréen (concernant en particulier les adverbes), propose un niveau sous-syntagmatique (« subphrasal ») en syntaxe qui combine des $\mathrm{X} 0$ et non des projections maximales : (i) certains mots (X0) peuvent être directement combinés en syntaxe, sans être dominés par une projection maximale ; (ii) certaines combinaisons de mots sont de niveau X0, alors que d'autres sont au moins de niveau X1 ; (iii) l'ordre des constituants X0 est plus contraint que celui des constituants X1 (ou plus), les premiers doivent être adjacents à la tête (qu'ils précèdent en coréen) tandis que les seconds peuvent permuter librement (en restant à gauche de la tête en coréen). Nos propositions transposent les différences de niveau de barre en termes de poids ${ }^{32}$. En effet, la représentation structurale rencontre des difficultés analogues à celle de SproatShih avec les données lexicales dans le problème qui nous occupe (et qui n'est pas l'objet du travail de Sells). Tous les A0 ne se comportent pas de la même manière. Or, il est difficile de forcer certains $\mathrm{A}$ (ou adverbes) à être dominés par une projection non lexicale, sans que tous aient la même possibilité. La différence de niveau est inopérante là où elle n'est pas liée à des différences de valence (ou de saturation). De même, on voit mal comment certains syntagmes modifiés ou 
coordonnés pourraient être sous-spécifiés quant au niveau de barre. Là encore, l'utilisation d'un trait apporte plus de souplesse.

En tant qu'elle introduit des différences de poids dans le lexique, notre proposition peut être rapprochée de la distinction de Cardinaletti et Starke (1994 = C \& S) entre formes « fortes » et formes « déficientes », si l'on considère nos adjectifs légers comme déficients (ou formes faibles). Bien que leur travail porte essentiellement sur les formes pronominales, ils notent une extension possible aux adjectifs, aux quantifieurs et aux adverbes, avec seulement des exemples des deux derniers [tout et bien, que nous analysons aussi comme des formes légères (Abeillé et Godard, 1998a) sont analysés comme déficients]. Nos adjectifs légers ont plusieurs propriétés communes avec celles des formes déficientes selon C \& S : (i) ils sont souvent homonymes ou synonymes de formes fortes (non légères pour nous) ; (ii) ils sont sujets (comme les pronoms faibles) à des phénomènes de sandhi (ou de restructuration prosodique) auxquels échappent les formes fortes ; (iii) ils apparaissent dans une position spéciale, non ouverte à tous les modifieurs dans le $\mathrm{GN}^{33}$. Il y a donc une convergence intéressante entre les deux propositions, faites de manière indépendante. Cependant, si l'on assimile les A légers à des formes faibles, il faut signaler une différence : nous ne pouvons interdire aux formes faibles dans leur ensemble d'être modifiées ou coordonnées puisque les A légers, prénominaux peuvent être modifiés (par certains adverbes) et coordonnés entre eux ; nous sommes conduits à distinguer deux types de modification et de coordination, dont l'un est compatible avec la déficience ${ }^{34}$. D'autre part, notre travail a l'avantage de donner un statut formel à la distinction, grâce à l'utilisation d'un trait, dans une grammaire qui utilise les structures de traits de manière systématique. Ce trait permet de faire sens des différences lexicales et syntagmatiques dans un système de contraintes sur l'ordre. Il permet également de garder sous-spécifiés les adjectifs mobiles non ambigus sémantiquement, alors que, dans la logique de $\mathrm{C} \& \mathrm{~S}$, on devrait systématiquement dédoubler les adjectifs mobiles (selon une paire faible/fort).

Nous terminons par la proposition la plus proche de la nôtre, celle de Sadler et Arnold (1994). Pour traiter l'ordre de l'adjectif et du N en anglais, ils proposent un trait à valeur binaire LEX présent sur les items lexicaux comme sur les syntagmes : (i) les mots sont [LEX +] ; (ii) certains syntagmes sont [LEX +], c'est-à-dire les coordinations de [LEX +] ou la modification d'un [LEX +] par un [LEX +] ; (iii) le schéma de modification comporte un accord tel que les A[LEX + ] modifient les N[LEX +], et inversement, les A[LEX-] modifient les N[LEX-] ; (iv) les $\mathrm{A}[\mathrm{LEX}+]$ précèdent les $\mathrm{N}$ têtes tandis que les $\mathrm{A}[\mathrm{LEX}-]$ les suivent.

Comme on le voit, nous gardons l'idée de base de ce travail, puisque nous faisons dépendre l'ordre de l'adjectif et du $\mathrm{N}$ d'un trait syntaxique. Nous avons modifié leur analyse en adoptant le trait de poids pour les raisons suivantes. D'abord, le trait LEX n'est pas adapté, puisque les classes d'items lexicaux, en particulier les A, sont divisés du point de vue des propriétés d'ordre, et les syntagmes seraient également divisés. On perd donc l'intuition qui lie le trait LEX aux mots (Pollard et Sag, 1987). D'autre part, nous voulons pouvoir noter le fait 
que les adjectifs après le $\mathrm{N}$ permutent avec les compléments, et, donc, dans notre hypothèse, se trouvent au même niveau. Le français n'offre donc pas l'accord proposé entre les traits de $\mathrm{A}$ et de $\mathrm{N}$ (un $\mathrm{N}[\mathrm{LEX}+$ ] ou léger est modifié par un $\mathrm{A}[\mathrm{LEX}-]$ ou non léger). Enfin, le trait de poids est plus adapté à la perspective que nous adoptons, dans laquelle l'ordre du $\mathrm{A}$ et du $\mathrm{N}$ sont vus comme un problème particulier de l'ordre des mots.

\section{Conclusion}

L'ordre relatif du $\mathrm{N}$ et du A épithète dépend à la fois des propriétés de l'item lexical et du type de syntagme auquel il appartient. Les premières ne se ramènent pas à de grandes classes morphologiques ou sémantiques, bien qu'il y ait des sous-généralisations intéressantes. Nous proposons un trait de poids (à valeur léger $v s$ non léger) qui est pris en compte par les contraintes de linéarisation. Ce trait est pertinent pour les mots et les syntagmes. Il peut capter les généralisations concernant les mots, en imposant des contraintes liées aux classes appropriées, tout en laissant sous-spécifiés les A mobiles. En contrastant les propriétés syntaxiques des A antéposés et postposés, nous montrons que l'antéposition met en jeu une combinaison directe ou quasi lexicale, tandis que la postposition implique une combinaison syntagmatique, directe (avec le N) ou indirecte (avec le GN). Notre proposition est que les A antéposés sont des mots « légers » (ou formes syntaxiquement faibles), et que l'antéposition de l'A épithète est précisément une des marques de sa déficience syntaxique (corrélée à son incapacité à projeter un syntagme complet), déficience qu'on retrouve avec certains adverbes antéposés au verbe infinitif (la classe de bien, mal, etc.).

Notre proposition est proche d'un certain nombre d'études récentes, touchant tant la position de l'épithète dans le GN que d'autres problèmes d'ordre (adverbes, compléments) ou la notion de déficience syntaxique (pronoms faibles). Il y a là une convergence intéressante sur les problèmes et les analyses. Notre travail a l'ambition d'inscrire la question de l'ordre du A et du $\mathrm{N}$ dans l'ensemble des problèmes d'ordre et d'en fournir une formalisation précise, et aisément transportable aux problèmes d'ordre dans des langues diverses.

\section{NOTES}

1. Cet article reprend partiellement l'analyse présentée dans Abeillé \& Godard (1998b). Il s'inscrit dans le projet d'une grammaire syntagmatique du français, auquel collabore Ivan Sag. Nous remercions pour leurs commentaires ou suggestions D. Arnold, J. Bresnan, R. Borsley, D. Corbin, D. Flickinger, G. Green, E. Hinrichs, S. Kahane, S. Lappin, D. Meurers, P. Miller, C. Pollard, F. Popowich, R. Kempson, L. Sadler, P. Sauzet, P. Sells, et tout particulièrement I.A. Sag.

2. Voir par exemple le panorama de Delomier (1981).

3. Ce choix s'oppose à celui que l'on trouve dans certains travaux transformationnels récents, illustré par exemple par Kayne (1994). 
4. La généralisation de Gougenheim (1939) selon laquelle « l'adjectif postposé a sa valeur propre tandis que l'adjectif antéposé a seulement une valeur appréciative » est une tendance souvent observée [voir aussi les observations de Blinkenberg (1928) concernant la valeur « affective » de l'adjectif antéposé]. Ces effets de sens, qui sont en dehors de notre travail, pourraient être traités par des contraintes pragmatiques additionnelles. L'hypothèse d'une différence sémantique systématique correlée à la position du $\mathrm{A}$ a été récemment défendue par Bouchard (1998). Malgré des exemples très intéressants, la tentative ne nous paraît pas vraiment convaincante [pour les subsectifs, voir la discussion de (4c), pour les intensionnels, voir les faits de coordination en (11)-(12), pour les A intersectifs non classifiants, la différence n'est pas perceptible, cf. un charmant jeune homme/un jeune homme charmant].

5. Cette bipartition prédicatif/non-prédicatif rejoint la différence souvent notée entre les A doués d'une sémantique «autonome» et ceux dont l'interprétation dépend de celle du N. Il existe un autre usage de la distinction, selon lequel un A prédicatif est celui qui peut être employé comme attribut. Les deux distinctions ne se recouvrent pas. Ainsi, certains non-prédicatifs (ordinaux et cardinaux, certains subsectifs) peuvent être attributs.

6. Selon la définition de Kamp (1975), les A qui satisfont au syllogisme (i) sont prédicatifs (ii), et les autres (iii) ne le sont pas :

(i) Tous les X's sont des Y's (ii) Les souris sont des rongeurs

(iii) Les éléphants sont des mammifères

Z est un AX

Minnie est une souris blanche

Donc, $\mathrm{Z}$ est un AY

Donc, Minnie est un rongeur blanc

Babar est un petit éléphant

Donc, Babar est un petit mammifère

7. Un petit éléphant est un éléphant et il est petit pour un éléphant.

8. Les classifiants (Milner, 1978) sont parfois appelés « objectifs », et les non-classifiants «subjectifs».

9. Pour les adjectifs de couleur, Wilmet note que $95 \%$ des occurrences sont après le $\mathrm{N}$ dans son corpus. Pour les $5 \%$ restants, il s'agit d'usages littéraires (de vertes frondaisons) qui représentent des emprunts à un système plus ancien de la langue, ou d'écarts par rapport au système que nous décrivons (vos rouges tabliers). Par ailleurs, nous ne considérons pas les adjectifs antéposables : pâle, sombre, clair comme des couleurs (mais comme dénotant une luminosité).

10. Les adjectifs dits relationnels ont généralement un emploi argumental et un emploi intersectif (les voyages présidentiels $=$ du président, ou $=$ de type présidentiel).

11. Nous distinguons ce cas de celui où la combinatoire entre l'adjectif antéposé et le $\mathrm{N}$ est figée, comme : grand-mère/père, petit fils/fille/enfant, petit ami, chaud lapin.

Nous traitons ces exemples comme des $\mathrm{N}$ composés lexicalisés, qui ne relèvent pas de notre propos.

12. Avec les subsectifs qui ont naturellement les deux positions, comme immense, les deux interprétations sont disponibles pour le A postposé : C'est un artiste immense (en tant qu'homme/ par sa taille/ou en tant qu'artiste/par son talent).

13. Certains adjectifs argumentaux sont meilleurs avant les GP :

(i) La production pétrolière du Maroc $/ *$ du Maroc pétrolière

(ii) La production marocaine de pétrole / de pétrole marocaine

14. Nous ne considérons pas ici les quelques adjectifs pouvant apparaître en initiale de GN (seul, feu).

15. En tant que modifieur indirect (de GN), l'épithète postposé peut être introduit par un marqueur explicite, ce que ne peut pas l'épithète antéposé :

(i) quelque chose de petit $v s$ un petit quelque chose

(ii) rien de simple $v s$ un simple rien 
16. Nous considérons une intonation non marquée : s'ils sont fortement accentués, ces adverbes perdent leurs propriétés spécifiques.

17. Elles se retrouvent aussi avec les quantifieurs «nus » (voir Abeillé \& Godard, 1998a). Comme nous l'a fait remarquer P. Sauzet, certains GN figés ne peuvent pas permuter non plus : mettre le feu à $\mathrm{X} / *$ mettre à $\mathrm{X}$ le feu.

18. Certains peuvent précéder l'infinitif (vraisemblablement, souvent, etc.) ou le verbe conjugué (vraisemblablement, demain, etc.) mais en s'adjoignant à $\mathrm{GV}$ (ou à la phrase), et non à V (voir Abeillé \& Godard, 1997 et A \& G).

19. Les compléments syntagmatiques (ou dans nos termes non légers) permutent aussi entre eux dans le GN : la volonté de lutter de Jean/de Jean de lutter. Des contraintes à base sémantique peuvent bien sûr s'ajouter. Par exemple, dans le GV, certains adverbes temporels précèdent les adverbes de manière (Les ter roristes ont immédiatement violemment riposté/* ont violemment immédiatement riposté). De même, un complément direct suit plus facilement un complément indirect quand il apporte une information nouvelle. Ce type de contraintes s'ajoute aux contraintes syntaxiques que nous regardons.

20. Bien que nous laissions de côté les syntagmes lourds, notre analyse se situe dans une perspective générale où ils ont leur place (et doivent suivre les non-lourds selon un ordre : léger < moyen < lourd, où les poids moyens ne sont ni lourds ni légers) [sur la « lourdeur », voir Wasow, 1996].

21. Voir Pollard et Sag $(1987,1994)$ et Abeillé (1993, chap. 3) pour une présentation du modèle.

22. La spécification du poids n'a pas à être faite pour chaque lexème indépendamment. Là où elle est contrainte, cette valeur peut être liée aux types morphologiques ou sémantiques dont les adjectifs héritent. On notera cependant qu'il s'agit en général d'une valeur par défaut. Ainsi, les adjectifs non prédicatifs sont (par défaut) légers, les adjectifs dérivés des participes sont (par défaut) non légers, et les adjectifs intersectifs classifiants sont non légers (contrainte dure).

23. Contrairement à la théorie X-barre, nous traitons les mots «nus » comme des constituants capables d'apparaître directement dans la construction syntaxique, même s'ils ne sont pas têtes. Alternativement, on pourrait dire que les mots (sans modifieur ni complément) sont dominés par un syntagme non branchant, à condition que le poids du syntagme soit identique à celui de la tête (il peut être léger).

24. B-Tête signifie branche tête, B-Non-Tête note l'ajout en (30a), les compléments et les ajouts en (30b).

25. Le signe U note l'unification et le signe D la disjonction (ou). Nous ne détaillons pas ici le syntagme-coordonné, qui est considéré comme sans tête (les conjonctions étant des marqueurs sur les constituants coordonnés).

26. Les types de syntagmes peuvent aussi spécifier le poids de leur tête : la tête du syntagme tête-compléments-ajout doit être légère, mais n'est pas contrainte dans les syntagmes tête-ajout et tête-spécifieur.

27. Le poids est un trait syntaxique, indépendant du poids phonologique (nombre de syllabes pour les mots), voir supra. Les A sont lexicalement spécifiés pour le poids en tant qu'épithètes. En tant qu'attributs, ils sont toujours non légers (ils peuvent permuter avec d'autres compléments et peuvent être extraits).

28. Nous laissons de côté l'ordre des adjectifs entre eux, qui dépend largement de leur classification sémantique. Comme le notent Sproat et Shih (1988), cet ordre semble plus contraint avant qu'après le nom : un gentil petit chien/* un petit gentil chien vs un chien noir méchant/un chien méchant noir. Cela n'est pas étonnant vu les contraintes plus fortes restreignant la mobilité des mots légers (voir $\mathrm{A} \& \mathrm{G}$ où nous montrons que, dans le $\mathrm{GV}$, les compléments légers sont ordonnés entre eux alors que les non légers ne le sont pas). 
29. Nous analysons les adverbes légers qui suivent $V$ comme des compléments, voir A \& G. Si l'on voulait les analyser comme des ajouts, il faudrait modifier la contrainte d'ordre pour restreindre son champ d'application. Les adverbes de négation (pas, jamais) sont légers ; ils modifient le GV infinitif, qu'ils précèdent, suivant la contrainte (38a). Ce sont les adverbes euxmêmes qui spécifient, grâce au trait MODIF, à quelles catégories ils peuvent s'adjoindre.

30. (39b) est une contrainte simplifiée : elle ne tient pas compte, en particulier, des adverbes non légers dont la position est libre dans le GV.

31. Il s'agirait alors de la formation de mots composés par des fonctionnements qui existent en syntaxe (qu'on peut appeler «incorporation» si on le désire), et non plus seulement de cas isolés (le bien-être, un va-et-vient). Cette analyse ferait sens des phénomènes de sandhi qui caractérisent les A prénominaux.

32. En ce qui concerne la proposition (i), voir la note 23.

33. Nous transposons l'analyse de Cardinaletti \& Starke, qui adoptent une optique transformationnelle ; pour eux, les formes faibles apparaissent dans une position « dérivée », tandis que les formes fortes apparaissent dans une position de base ou extraite.

34. Cardinaletti \& Starke proposent aussi une corrélation entre propriétés sémantiques et déficience : les déficients ont une sémantique moins autonome. Pour les A, si l'on assimile sémantique " non autonome » à interprétation non prédicative, il y a bien convergence avec la déficience (ou légèreté), mais avec des exceptions, puisque certains A subsectifs peuvent être postnominaux, donc non légers ou forts (voir immense, etc., voir 1.3), et que des A intersectifs (donc avec une interprétation autonome) peuvent être prénominaux. Pour les adverbes, il n'y a pas de corrélation satisfaisante entre le poids/la déficience et la sémantique. D'autre part, dans la logique de Cardinaletti \& Starke, on s'attend à une tripartition des formes adjectivales, certaines étant plus déficientes que d'autres. La prise en compte d'une troisième valeur (super-léger ou poids plume) dans notre système pourrait expliquer pourquoi certains adjectifs prénominaux, même coordonnés ou modifiés, ne peuvent se postposer (les cardinaux, les ordinaux, les intensionnels de type gros fumeur ou grand travailleur).

\section{RÉFÉRENCES}

AbeILLÉ, Anne (1993). Les Nouvelles Syntaxes: grammaires d'unification et analyse du français, Paris : Armand Colin.

AbeILlÉ, Anne ; Godard, Danièle (1997). The Syntax of French Negative Adverbs.Dans D. Forget, P. Hirschbühler, F. Martineau, M-L. Rivero (eds), Negation and Polarity, syntax and semantics : 1-27. Amsterdam: J. Benjamins.

Abeillé, Anne ; GoDARD, Danièle (1998a). A Lexical account of quantifier floating.Dans A. Kathol, J-P. Koenig et G. Webelhuth (eds), Lexical and Constructional Aspects of Linguistics Explanation: 81-86.Stanford : CSLI.

Abeillé, Anne ; GodARD, Danièle (1998b). French word Order and lexical Weight.Dans R. Borsley (ed), Syntactic Categories, Syntax and Semantics, Academic Press, New York, à paraître.

Blinkenberg, Andreas (1928). L'Ordre des mots en français moderne. Copenhague : Munksgaard.

BOUCHARD, Denis (1998). The distribution and interpretation of adjectives in French : a consequence of bare phrase structure, Probus.

Cardinaletti, Anna ; STARKe, Michael (1994). The Typology of structural deficiency : on the three grammatical classes. Working papers in linguistics, 4-2, Université de Venise ; à paraître dans H. Riemsdijk (ed), Clitics in the languages of Europe, Berlin : Mouton de Gruyter. 
Cinque, Guglielmo (1990). Agreement and Head-to-Head movement in the Romance Noun Phrase. Actes LSRL XX, University of Ottawa.

DelbeCQue, Nicole (1990). Word order as a reflexion of alternate conceptual construals in French and Spanish. Similarities and divergences in adjective position. Cognitive Linguistics : 349-416.

Delomier, Dominique (1980). La place de l'adjectif en français, bilan des points de vue et théories du $\mathrm{Xx}^{\mathrm{e}}$ siècle. Cahiers de lexicologie, 37: 5-24.

Forsgren Mat (1978). La Place de l'adjectif épithète en francais contemporain, étude quantitative et sémantique, Stockholm : Almqvist \& Wiksell.

Gazdar, Gerald ; Klein, Ewan ; Pullum, Geoffrey ; Sag, Ivan A. (1985). Generalized Phrase Structure Grammar, Cambridge: Cambridge University Press.

Gougenheim Georges (1939). Le Système grammatical de la langue française, Paris : Editions d'Artrey.

Grevisse, Maurice (1988). Le Bon usage, Louvain : Duculot, $12^{\mathrm{e}}$ édition (revue par A. Goosse).

Kamp, Hans (1975). Two theories about adjectives. Dans E. Keenan (ed), Formal semantics of natural language : 123-155. Cambridge : Cambridge University Press.

KASPER, Robert (1994). Adjuncts in the Mittelfeld. in J. Nerbonne et al. (eds), German in HeadDriven Phrase Structure Grammar :39-69. Stanford : CSLI.

KAYNE, Richard (1994). The Antisymetry of Syntax, Cambridge: MITPress.

Miller, Philip (1991). Clitics and Constituents in Phrase Structure Grammar, Thèse de l'université d'Utrecht [publié, New York : Garland, 1992].

Miller, Philip ; Pullum, Geoffrey ; Zwicky, Arnold (1997). The principle of phonology-free syntax: four apparent counterexamples in French. Journal of Linguistics, 33 : 67-90.

MiLner, Jean-Claude (1978). De la syntaxe à l'interprétation, Paris : Le Seuil.

NøLKE, H. (1996). Où placer l'adjectif épithète ? Focalisation et modularité. Langue française, $111: 38-57$.

PollaRd, Carl ; S AG, Ivan A. (1987). Information-based Syntax and Semantics, CSLI series, distr. University of Chicago Press.

Pollard, Carl ; SAG, Ivan A. (1994). Head-driven Phrase Structure Grammar, Stanford : CSLI and Chicago: Chicago University Press.

Rouveret, Alain (1994). Syntaxe du gallois, Principes et typologie, Paris : Editions du CNRS.

SAdLeR, Louisa ; ARnold, Doug (1994). Prenominal Adjectives and the phrasal/lexical distinction, Journal of Linguistics $30: 187-226$.

SAG, Ivan A. (1997). English relative clause constructions, Journal of Linguistics 33.2: 431-484.

Sells, Peter (1994). Sub-phrasal Syntax in Korean, Language Research, Seoul 30 : 351-386.

SPROAT, Richard ; SHIH, Chilin (1988). Prenominal adjectival ordering in English and Mandarin, NELS.

VALoIs, Daniel (1991). The internal syntax of DPand adjective placement in French and English. NELS: $367-381$.

WASOW, Tom (1996). « Remarks on Grammatical Weight ». Ms. Stanford University.

WAUGH, Linda (1977). A semantic analysis of word order. Leiden.

WiLLIAMs, Edwins (1982). Another argument that passive is transformational, Linguistic Inquiry: 160-162.

WiLmet, Marc (1981). La place de l'épithète qualificative en français contemporain ; étude grammaticale et stylistique. Revue de linguistique romane, $45: 17-73$. 


\section{Abstract}

We suggest an explanation of the position of the attributive adjective with respect to the Noun in French, based on the notion of syntactic Weight. In our approach, adjectives belonging to certain morphological or semantic subclasses are marked as being "lite weight", and must be preposed, except if they are modified or conjoined. The notion of liteness also accounts for the impossibility to prepose full adjectival phrases (with complements or phrasal modifiers).

\section{KEYWORDS}

Attributive adjective, French, Syntactic deficiency, HPSG, Word order, Weight. 